Ephemeride des Planeten (349) Dembowska für die vierte Opposition.

I $2^{\text {h }}$ M. Z. Berlin.

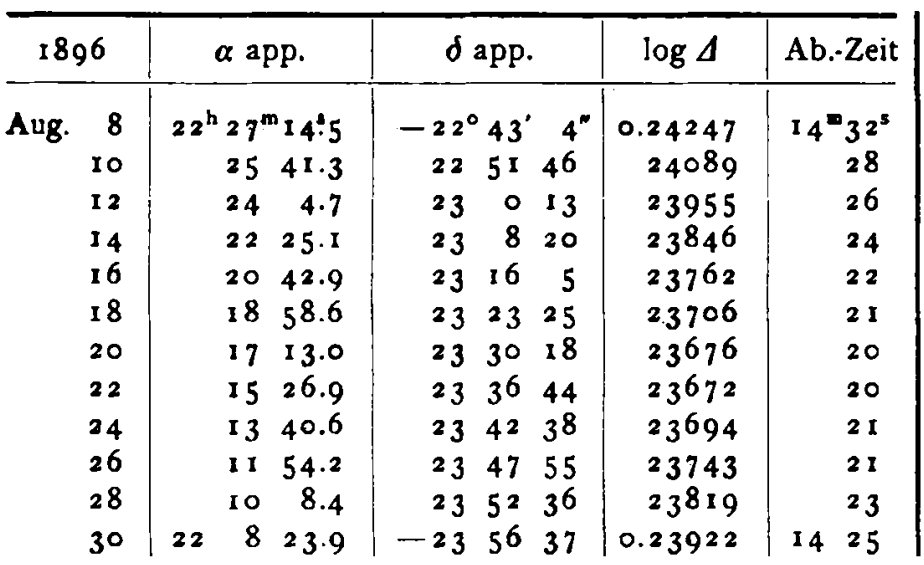

\begin{tabular}{|c|c|c|c|c|c|c|c|c|}
\hline 1896 & \multicolumn{3}{|c|}{$\alpha$ app. } & \multicolumn{3}{|c|}{$\delta$ app. } & $\log A$ & Ab.-Zeit \\
\hline ug. 30 & $22^{h}$ & & $23: 9$ & $-23^{\circ}$ & $56^{\prime}$ & $37^{\prime \prime}$ & 0.23922 & $14^{m} 25^{s}$ \\
\hline ept. I & & & 41.1 & 23 & 59 & 58 & 2405 I & 28 \\
\hline 3 & & & 0.3 & 24 & 2 & 42 & 24204 & 31 \\
\hline 5 & & & 22.1 & 24 & 4 & 46 & $243^{8 I}$ & 34 \\
\hline 7 & & & 46.8 & 24 & 6 & 5 & $245^{82}$ & $3^{8}$ \\
\hline 9 & 22 & 01 & 15.2 & 24 & 6 & 42 & 24807 & 43 \\
\hline II & 215 & 84 & 48.1 & 24 & 6 & $3^{1}$ & 25054 & 48 \\
\hline 13 & & 72 & 25.4 & 24 & 5 & 37 & 25323 & 53 \\
\hline 15 & & & 7.0 & 24 & 4 & 3 & 25611 & 1459 \\
\hline 17 & & 45 & 53.6 & 24 & I & 47 & $259 \times 8$ & I 56 \\
\hline 19 & 5 & 34 & $45 \cdot 5$ & 23 & $5^{8}$ & 47 & 26243 & I 3 \\
\hline 21 & 215 & 324 & 43.2 & -23 & 55 & 7 & 0.26584 & 1520 \\
\hline
\end{tabular}

Opposition in AR. Aug. 24; Grösse 9:4. Reduction auf $1855.0: \Delta \alpha-2^{m} 16^{s}, \Delta \delta-12: 2$.

Die Ephemeride beruht auf den Elementen des Berliner Jahrbuchs. Nur die mittlere Bewegung wurde un o:8844 vermindert, wodurch die Abweichungen $B-R$ der dritten Opposition zum Verschwinden gebracht werden. Einer Aenderung von $\pm 10^{5}$ in $A R$. entspricht für den An-

Karlsruhe 1896 Juli $3 \mathbf{I}$. fang der Ephemeride eine solche von $\pm 66^{\prime \prime}$ in Decl. $\mathrm{Da}$ der Planet in der dritten Opposition nur sehr wenig beobachtet wurde, sind Beobachtungen in dieser Erscheinung um so erwlinschter.

\title{
Ueber die Wiederkehr des Cometen 1890 VII (Spitaler).
}

Die Bearbeitung dieses Cometen für die Zeit seiner ersten Erscheinung und der.. bevorstehenden. Wiederkehr wurde in den Sitzungsberichten der Kaiserlichen Akademie der Wissenschaften in Wien niedergelegt und wird demaächst veröffentlicht werdea. Das nächste Perihel erreicht der Comet am 11. März 1897, doch gelangt er zur Erde bereits im September und October l. J. in die günstigsten Sichtbarkeitsverhältnisse (Opposition in AR. am I3. Sept. 1896), weshalb ich mir erlaube die nachstehende Aufsuchungsephemeride rechtzeitig bekannt zu geben.

Es wäre von grossem Werth, wenn der Comet bei der gegenwärtigen Erscheinung wieder aufgefunden würde, da dies bei der Wiederkehr im Jahre 1903 wegen un. günstiger Stellung zur Erde nicht möglich sein wird; erst Ende 1909 werden die Sichtbarkeitsverhältnisse wieder sehr günstige sein. Ich wäre den Herren Beobachtern mit grösseren Fernrohren zu Dank verpflichtet, wenn sie sich an der Hand der folgenden Ephemeride die Aufsuchung des Cometen angelegen sein liessen. Ich bitte insbesondere auch die Herren Astrophotographen durch gelegentliche Aufnahmen der betreffenden Himmelsgegend vielleicht die Auffindung zu ermöglichen. Als Einheit der Helligkeit ist in der Aufsuchungsephemeride jene angenommen, welche der Comet zur Zeit seiner Entdeckung besass, wo er eine Coma von ungefahr $1 / 2^{\prime}$ Durchmesser und einen deutlichen Kern 13. Grösse hatte.

Die Abweichung des Cometen von der Ephemeride dürte im ungünstigsten Fall zu Anfang September $\Delta \alpha=$ $\pm 5^{\mathrm{m}} 4^{8^{\mathrm{s}}}, \Delta \delta= \pm 43^{\circ}$, zu Anfang November $\Delta \alpha= \pm 4^{\mathrm{m}} 35^{\mathrm{s}}$, $\Delta \delta= \pm 35^{\circ}$ betragen.

Die wahrscheinlichsten Elemente auf 1896.0 übertragen lauten:

$$
\begin{aligned}
& \text { Epoche }{ }_{1} \delta 96 \text { Sept. I.5 M. Z. Berlin. } \\
& M=330^{\circ} 29^{\prime} 39^{\prime \prime} 10 \\
& \pi^{\prime}=6024 \quad 54.95 \\
& \Omega^{\prime}=1630 \quad 9.38 \\
& i^{\prime}=334057.84 \\
& \varphi=2876.60 \\
& \mu=556.7675
\end{aligned}
$$

\begin{tabular}{|c|c|c|c|c|c|}
\hline 1896 & $\alpha$ app. & $\delta$ app. & $\log r$ & $\log 4$ & H \\
\hline Sept. I & $\mid \begin{array}{rrr}23^{\mathrm{h}} & 37^{\mathrm{m}} & 55^{\mathrm{s}} .9 \\
& 37 & 4.9 \\
23 & 3^{6} & 12.8\end{array}$ & $\left|\begin{array}{rrr}-23^{\circ} & 34^{\circ} & 50^{\prime \prime} \\
23 & 39 & 28 \\
-23 & 43 & 5^{6}\end{array}\right|$ & $0.3^{806}$ & 0.1552 & 0.23 \\
\hline
\end{tabular}

\begin{tabular}{|c|c|c|c|c|c|c|}
\hline 1896 & & $\alpha$ app. & $\delta$ app. & $\log r$ & $\log \Delta$ & $\mathrm{H}$ \\
\hline Sept. & $\begin{array}{l}4 \\
5 \\
6\end{array}$ & 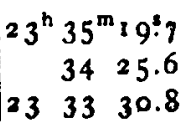 & $\begin{array}{rrr}-23^{\circ} 4^{8} & 15 \\
23 & 5^{\prime 2} & 23 \\
-23 & 5^{6} & 20\end{array}$ & $0.377 \mathrm{I}$ & 0.1488 & 0.24 \\
\hline
\end{tabular}

Rechtwinklige Aequatorcoordinaten.

$$
\begin{aligned}
& x=[9.994543] r \cdot \sin \left(v+147^{\circ} 45^{\prime} 41^{\prime \prime} .4\right) \\
& y=[9.927834] r \cdot \sin (v+633036.4) \\
& z=[9.743975] r \cdot \sin (v+435445.6)
\end{aligned}
$$

Ephemeride für I $^{\text {h }}$ M. Z. Berlin. 


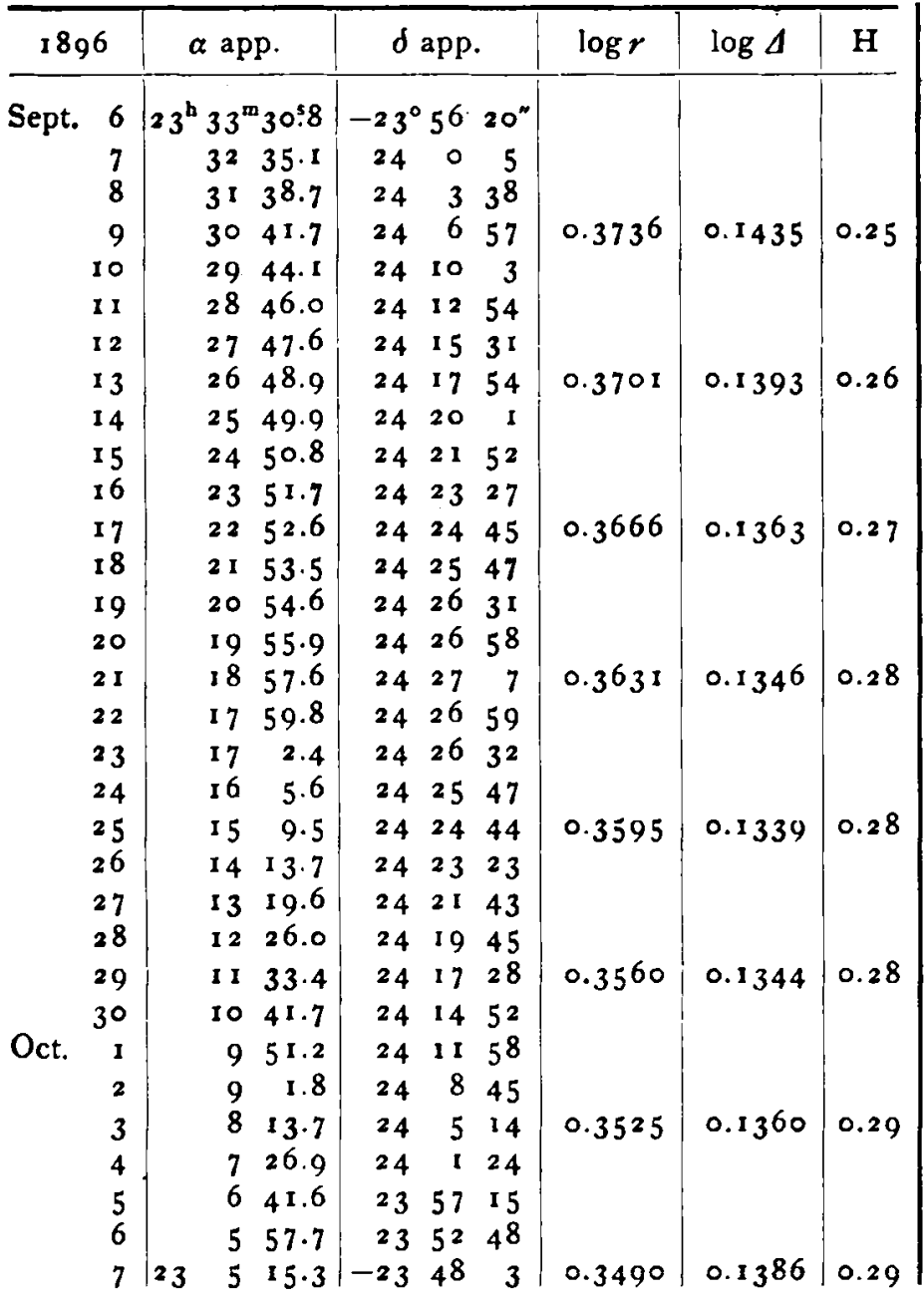

Prag, k. k. Sternwarte, 1896 Aug. 2.

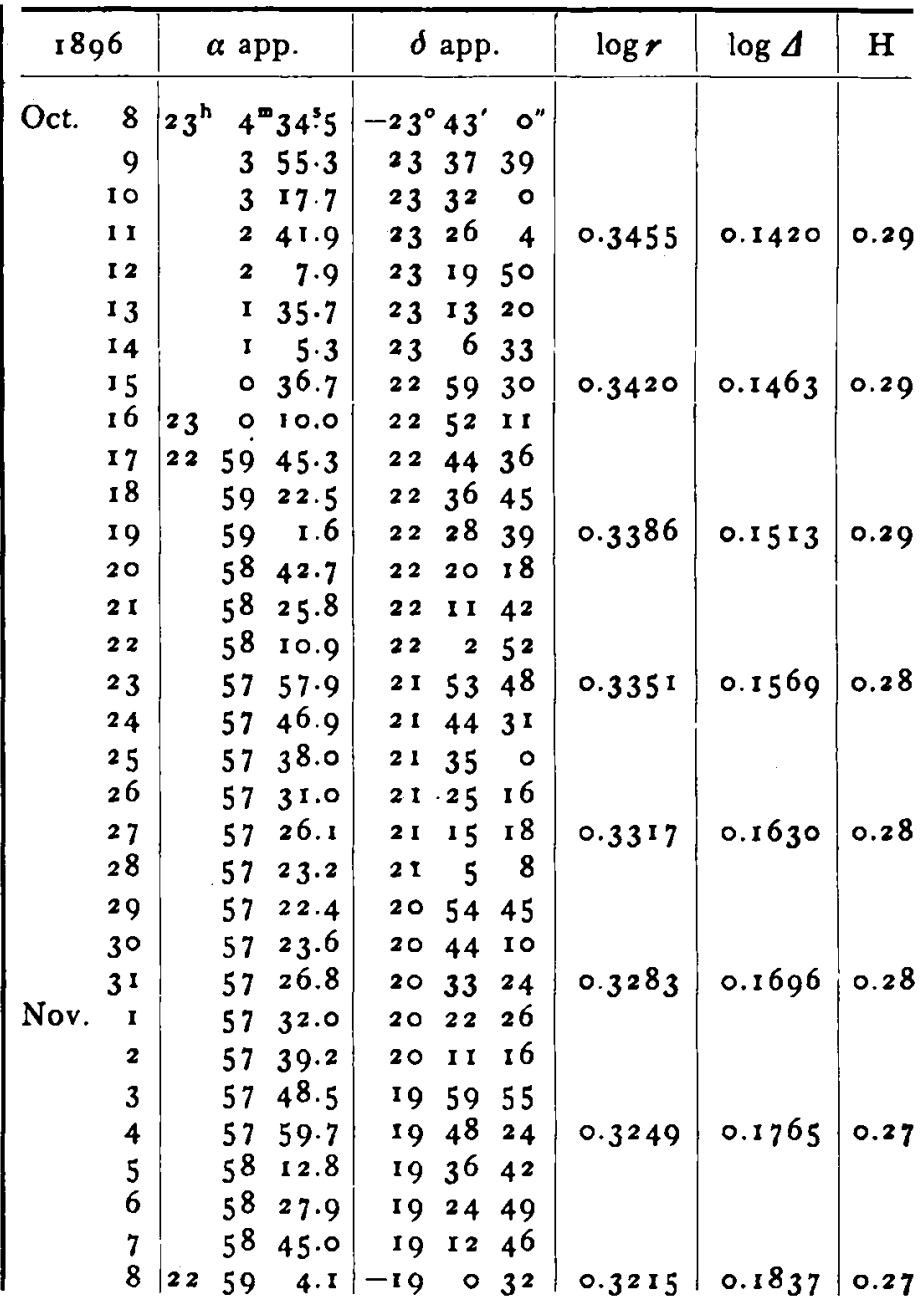

Dr. R. Spitaler.

\section{Beobachtung des Planeten (304) Olga. *)}

Ich habe den Planeten auf einer Platte vom 3. August gefunden und heute am $12 \mathrm{z}$. Refractor beobachtet; die Correction der Ephemeride des Herrn Berberich in A. N. $33^{6} 5$ beträgt $+7^{\mathrm{m}} 26^{\mathrm{s}}+6: 8$. Der Planet ist $1 \mathrm{r}{ }^{\mathrm{m}} 5$.

Berlin, Urania, I 896 Aug. 7.

Gustav Witt.

*) Eine von Herrn A. Berberich am 10. d. Mts. eingegangene verbesserte Ephemeride des Planeten kann wegen Raunmangels erst in der nächsten Nummer der A. N. Aufnahme finden. Einige Oerter derselben sind mehreren Sternwarten brieflich mitgetheilt worden. Kr.

\section{Sonnenfinsterniss 1896 Aug. 8.}

In Vadsö ist es nach einer telegraphischen Mittheilung von Prof. Geelmuyden leider trübe gewesen. Aus Pulkowa telegraphirt Herr Lindemann: \Amurstation Beobachtung durch leichte Wolken gelungen; in Pulkowa Austritt beobachtet. In Berlin (kgl. Sternwarte) wurde der Austritt beobachtet; in Heidelberg Regen; in Kiel zuerst klar, später trat die Sonne hinter eine Wolkenbank, die die Beobachtung des Austrittes verhinderte.

Kr.

Inhalt zu Nr. 3370. W. Doberck. On the orbit of $\eta$ Coronae Borealis. I 53. - E. C. Pickering. Stars having peculiar spectra. I69. - W. F. Denning. The belts and spots on Saturn. 171. - Beobachtungen der Bedeckung des Jupiter durch den Mond 1896 Juni 14. 171. - F. Ristenpart. Ephemeride des Planeten (349) Dembowska für die vierte Opposition. 173. - R. Spilaler. Ueber die Wiederkehr des Cometen 1890 VII (Spitaler). I73. - G. Witt. Beobachtung des Planeten (304) Olga. 175. - Sonnenfinsterniss 1896 Aug. 8. 175. 